\title{
On Performance of Weighted Fusion Based Spectrum Sensing in Fading Channels
}

\author{
Srinivas Nallagonda, Shravan Kumar Bandari, Sanjay Dhar Roy, and Sumit Kundu \\ Department of ECE, NIT Durgapur, West Bengal, India \\ Correspondence should be addressed to Srinivas Nallagonda; srinivas.nallagonda@gmail.com
}

Received 21 May 2013; Revised 7 October 2013; Accepted 20 October 2013

Academic Editor: Wuliang Yin

Copyright ( 2013 Srinivas Nallagonda et al. This is an open access article distributed under the Creative Commons Attribution License, which permits unrestricted use, distribution, and reproduction in any medium, provided the original work is properly cited.

Cognitive radio (CR) is a promising technology for efficient utilization of underutilized spectrum since it is able to detect the occupancy of primary users (PUs) in the different parts of the spectrum. As the sensing channel uncertainties limit the reliability of the spectrum sensing decision, cooperation among multiple CR users is often used to improve the spectrum sensing decision. In this paper, the performance of single CR user based spectrum sensing and cooperative CR user based spectrum sensing (CSS) has been assessed in several channels such as AWGN, log-normal, Hoyt (or Nakagami-q), Rayleigh, Rician (or Nakagami-n), Nakagami- $m$, and Weibull channels. The performance of two spectrum sensing schemes based on assigning weights to CR users such as (a) weighting according to sensing channel preference and (b) weighting according to the value of decision statistic is evaluated. The performance comparison between two weighting schemes under several fading channels has been made. The performance of proposed CSS has been illustrated through complementary receiver operating characteristics (CROC) for different fading channels. The effects of weighting factors $\left(k\right.$ and $\left.R_{f}\right)$ on overall missed detection performance are shown. The performance of CSS with ORlogic fusion as a special case is also presented for comparison purpose.

\section{Introduction}

Cognitive radio (CR) techniques have been proposed to overcome spectrum scarcity by exploiting underutilized spectrum [1]. It allows the CR users to share the spectrum with primary users (PUs) by opportunistic access. Cognitive radio is defined as a wireless radio device that can adapt to its operating environment through sensing in order to facilitate efficient communications. The CR user can use the spectrum only when it does not create any disturbance or interference to PUs. Thus sensing of vacant spectrum is very important for successful operation of cognitive radio network. Due to the severe multipath fading, a CR may fail to notice the presence of the PU. Therefore, spectrum sensing is an important aspect of CR technology since it needs to sense the PUs accurately and quickly $[2,3]$. Spectrum sensing is a hard task because of shadowing, fading, and time-varying nature of wireless channels [4]. The performance of single CR user based spectrum sensing has been studied in several channels such as additive white Gaussian noise (AWGN), lognormal shadowing, Rayleigh, Nakagami- $m$, and Weibull in
[5] where the Nakagami- $m$ and Weibull distribution provide flexibility in describing the fading severity of the channel and consider special cases such as the well known Rayleigh fading for a certain value of the fading parameter. Cooperative spectrum sensing (CSS) improves the detection performance where all CRs sense the PU individually and send their sensing information in the form of 1-bit binary decisions ( 1 or 0 ) to fusion center (FC) $[6,7]$. The hard decision fusion rules such as OR-logic, AND-logic, and Majority logic can be performed at FC to make the final decision regarding whether the PU is present or not. The performance of CSS using EDs has been studied in channels such as log-normal shadowing, Hoyt (or Nakagami-q), Rayleigh, Nakagami- $m$, Rician (or Nakagami-n), and Weibull in [8-11]. Hoyt distribution [12-14], also known as Nakagami- $q$ distribution ( $q$ being the fading severity parameter), allows us to span the range of fading distribution from one-sided Gaussian $(q=0)$ to Rayleigh fading $(q=1)$ and is used extensively for modeling channels which are more severe than Rayleigh fading wireless links. Rician distribution also known as Nakagami- $n$ distribution is used extensively for modeling less severe 
than Rayleigh fading wireless links. Weibull fading has been proved to exhibit excellent fitting for indoor [15] and outdoor [16] environments. Comparison among hard decision fusion rules for the case of CSS has been investigated in a Suzuki fading channel [17]. In most of the existing work on CSS, each CR is treated in the same manner, while fusing decisions from a number of participating CRs, through the sensing channel of the CRs, may be under fading with different level of severity. Thus we require a scheme where decisions from CRs are assigned appropriate weights. The weighted cooperative spectrum sensing (WCSS) scheme performance has been investigated in [18-21].

However, the existing works examined the CSS using different weighting schemes in log-normal shadowing and Rayleigh and Nakagami- $m$ fading channels only [22]. In this paper, we extend performance analysis of CSS in Hoyt, Rician and Weibull fading channels. We note the analytical expression for probability of detection at CR level in Rayleigh and Nakagami- $m$ fading channels [2]. We develop a simulation test bed to assess the probability of detection at CR equipped with energy detector under several types of fading in sensing channel. Further we compare results (specifically, Rayleigh and Nakagami-m) obtained via our simulation model with the results obtained using the expressions developed in [2]. Thus we verify our simulation testbed. The performance of single CR user and cooperative CR user based spectrum sensing has been assessed in terms of complementary receiver operating characteristics (CROC) in various fading channels. The performance comparison of CSS among fading channels using two weighting schemes, namely, weighting based on channel preference and weighting based on decision statistics, has been evaluated. The performance of weighting schemes is also compared with the performance of CSS without weighting (OR-logic fusion). The effects of weighting factors on overall missed detection performance at FC are also highlighted in this paper. More precisely, our contributions in this paper are as follows:

(i) evaluation of cooperative spectrum sensing (CSS) using two weighted based schemes;

(ii) performance evaluation has been extended in Hoyt, Rician and Weibull fading channels in addition to Rayleigh, Nakagami- $m$, and log-normal shadowing in sensing channel;

(iii) the performance in several fading channels as mentioned above has been compared;

(iv) CROC has been obtained for CSS as well as single CR based sensing in several fading channels;

(v) the weighting schemes based CSS are compared with conventional OR-logic based fusion without weighting;

(vi) a simulation test bed has been developed in MATLAB and validated to evaluate the performance as indicated above.

The rest of the paper is organized as follows. In Section 2, the system model under consideration is described and we briefly describe the probabilities of detection and of false

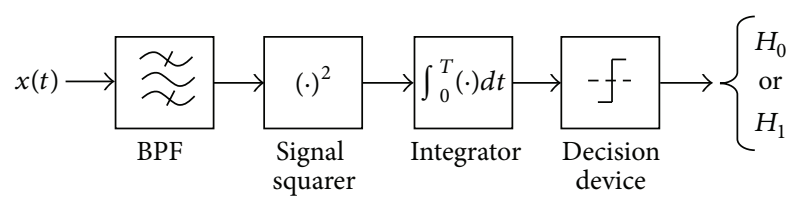

FIGURE 1: Block diagram of an energy detector.

alarm over additive white Gaussian noise (AWGN) channel, shadowing and fading channels, and weighting schemes. Our simulation model is presented in Section 3. Results and discussions are presented in Section 4. Finally we conclude in Section 5.

\section{System Model}

We consider a network of $N$ cognitive radio (CR) users, one primary user (PU), and one fusion center (FC). A CR user, which is using an energy detector (ED) with a detection threshold $(\lambda)$, makes hard binary decision (either "1" or bit "0") over fading or shadowing sensing channel. All CR users use the same threshold $(\lambda)$. The distance between any two CR users is less than the distance between a PU and a CR user or the distance between a CR user and the FC. Each CR user is having one ED as shown in Figure 1.

An energy detector receives a signal $x(t)$ as defined below at input and gives a binary decision regarding the presence or absence of the PU. The received signal $x(t)$ at $i$ th CR user can be represented as

$$
x_{i}(t)= \begin{cases}n_{i}(t), & H_{0}, \\ h_{i} s(t)+n_{i}(t), & H_{1},\end{cases}
$$

where $h_{i}$ is the sensing channel gain of $i$ th CR user. The noise $n_{i}(t)$ is modeled as a zero-mean white Gaussian random process and $H_{1}$ and $H_{0}$ are the two hypotheses associated with the presence and absence of a PU. The noise energy at $i$ th CR user can be approximated over the time interval $(0, T)$, as $[2,3]$

$$
\int_{0}^{T} n_{i}^{2}(t) d t=\frac{1}{2 W} \sum_{j=1}^{2 u} n_{i, j}^{2},
$$

where $u$ is the time-bandwidth product and

$$
n_{i, j} \sim N\left(0, N_{01} W\right), \quad \forall j .
$$

The decision statistic at $i$ th $\mathrm{CR}$ user, $Y_{i}$, can be written as

$$
Y_{i}=\sum_{j=1}^{2 u} n_{i, j}^{\prime 2}
$$

where $n_{i, j}^{\prime}=n_{i, j} / \sqrt{N_{01} W} . Y_{i}$ can be viewed as the sum of the squares of $2 u$ standard Gaussian variates with zero mean and unit variance. Therefore, $Y_{i}$ follows a central chi-square $\left(\chi^{2}\right)$ distribution with $2 u$ degrees of freedom. The same approach can be applied when the signal $s(t)$ is present, by replacing each of $n_{i, j}$ with $n_{i, j}+s_{j}$ where $s_{j}=s(j / 2 W)$ for a given $h_{i}$. 
Thus, the decision statistic $Y_{i}$ conditioned on $h_{i}$ will have a noncentral Chi-square $\chi^{2}\left(2 \gamma_{i}\right)$ distribution with $2 u$ degrees of freedom and a noncentrality parameter $2 \gamma_{i}[2,3]$. The actual statistics of $Y_{i}$ have to be found appropriately by averaging the statistics with probability density function (PDF) of fading coefficient $\left(h_{i}\right)$.

2.1. Analysis of Detection and False Alarm Probabilities. In this section we study probability of detection and probability of false alarm in nonfaded, that is, AWGN channel and faded environments. The probabilities of detection and false alarm for non-faded case are presented in Section 2.1.1, while the same parameters are presented for a general case of fading in Section 2.1.2.

2.1.1. Non-Fading Environment (AWGN Channel, i.e., $h_{i}=1$ ). In nonfading environment, that is, when the sensing channels are corrupted by AWGN only, the probabilities of detection and false alarm at $i$ th CR user are given by the following formulas $[2,5-7]$ :

$$
\begin{gathered}
P_{d, i}=P\left(Y_{i}>\lambda \mid H_{1}\right)=Q_{u}\left(\sqrt{2 \gamma_{i}}, \sqrt{\lambda}\right), \\
P_{f, i}=P\left(Y_{i}>\lambda \mid H_{0}\right)=\frac{\Gamma(u, \lambda / 2)}{\Gamma(u)},
\end{gathered}
$$

where $\Gamma(\cdot, \cdot)$ is the incomplete gamma function [23] and $Q_{u}(\cdot, \cdot)$ is the generalized Marcum Q-function [24]. For simplicity, identical average SNRs $\left(\gamma_{i}=\bar{\gamma} ; \forall i\right)$ have been assumed for all CR users. If the signal power is unknown, we can first set the false alarm probability $\left(P_{f}\right)$ to a specific desired level. For a given desired level of $P_{f}$, the threshold $(\lambda)$ of energy detector is set which determines the detection probability $\left(P_{d}\right)$ along with instantaneous $\operatorname{SNR}(\gamma)$ of sensing channel as given in (5). As expected, $P_{f}$ is independent of $\gamma_{i}$ for all $i$ since under $H_{0}$ there is no PU signal present.

2.1.2. Fading Environment. When $h$ is varying due to fading, (5) gives the probability of detection as a function of the instantaneous $\operatorname{SNR}(\gamma)$. In this case, the average probability of detection $\left(\bar{P}_{d}\right)$ may be derived by averaging (5) over fading statistics [6],

$$
\bar{P}_{d}=\int_{0}^{\infty} Q_{u}(\sqrt{2 \gamma}, \sqrt{\lambda}) f_{\gamma}(\gamma) d \gamma,
$$

where $f_{\gamma}(\gamma)$ is the PDF of SNR under fading. The expression for $P_{f}$ given in (6) remains the same for fading case due to independency of $\gamma$.

In the following subsequent sections, we study briefly various statistical models of several fading channels such as log-normal shadowing, Hoyt (Nakagami-q), Rayleigh, Rician, Nakagami- $m$, and Weibull fading channels.

2.2. Log-Normal Shadowing Channel. The linear channel gain may be modeled by Log-normal random variable $e^{X}$, where $X$ is a zero-mean Gaussian random variable with variance $\sigma^{2}$. Log-normal shadowing is usually characterized in terms of its dB-spread, $\sigma_{\mathrm{dB}}$, which is related to $\sigma$ by $\sigma=0.1 \ln (10) \sigma_{\mathrm{dB}}[6]$.
2.3. Hoyt or Nakagami-q Fading Channel. We assume that the sensing channel between PU and CR user is Hoyt faded. Hoyt or Nakagami- $q$ distribution is generally used to characterize the fading environments that are more severe than Rayleigh fading. The PDF of $\gamma$, that is, $f_{\gamma}(\gamma)$, may be defined as $[13,14]$

$$
f_{\gamma}(\gamma)=\frac{1}{\sqrt{p}} \exp \left(-\frac{\gamma}{p \bar{\gamma}}\right) I_{0}\left(\frac{\gamma \sqrt{1-p}}{p \bar{\gamma}}\right) ; \quad \gamma \geq 0,
$$

where

$$
p=\frac{4 q^{2}}{\left(1+q^{2}\right)^{2}} ; \quad 0 \leq p \leq 1
$$

where $q$ is the fading severity parameter. The average $P_{d}$ in this case, $\bar{P}_{d \text {, Hoyt }}$ can now be evaluated by substituting $f_{\gamma}(\gamma)$ from (8) to (7).

2.4. Rayleigh Fading Channel. If the signal amplitude follows a Rayleigh distribution, then the SNR $\gamma$ follows an exponential PDF given by [11]

$$
f_{\gamma}(\gamma)=\frac{1}{\gamma} \exp \left(-\frac{\gamma}{\bar{\gamma}}\right) ; \quad \gamma \geq 0
$$

The average $P_{d}$ in this case, $\bar{P}_{d \text {, Ray }}$, can be evaluated by substituting (10) in (7):

$$
\begin{aligned}
\bar{P}_{d, \text { Ray }}=\exp \left(-\frac{\lambda}{2}\right) \sum_{k=0}^{u-2} & \frac{1}{k !}\left(\frac{\lambda}{2}\right)^{k}+\left(\frac{1+\bar{\gamma}}{\bar{\gamma}}\right)^{u-1} \\
\times & \left(\exp \left(-\frac{\lambda}{2(1+\bar{\gamma})}\right)-\exp \left(-\frac{\lambda}{2}\right)\right. \\
& \left.\times \sum_{k=0}^{u-2} \frac{1}{k !}\left(-\frac{\lambda \bar{\gamma}}{2(1+\bar{\gamma})}\right)^{k}\right) .
\end{aligned}
$$

2.5. Rician Fading Channel. If the signal strength follows a Rician distribution, the PDF of $\gamma$ will be [2]

$$
\begin{aligned}
f_{\gamma}(\gamma)= & \frac{K+1}{\bar{\gamma}} \exp \left(-K-\frac{(K+1) \gamma}{\bar{\gamma}}\right) I_{0} \\
& \times\left(2 \sqrt{\frac{K(K+1) \gamma}{\bar{\gamma}}}\right), \quad \gamma \geq 0,
\end{aligned}
$$

where $K$ is the Rician factor. The average $P_{d}$ in the case of a Rician channel, $\bar{P}_{d \text {, Ric }}$, is then obtained by substituting (12) in (7). The resulting expression can be solved for $u=1$ using [24, Equation (45)] to yield

$$
\left.\bar{P}_{d, \text { Ric }}\right|_{u=1}=Q\left(\sqrt{\frac{2 K \bar{\gamma}}{K+1+\bar{\gamma}}, \sqrt{\frac{\lambda(K+1)}{K+1+\bar{\gamma}}}}\right) .
$$

For $K=0$, this expression reduces to the Rayleigh expression with $u=1$ [2]. 
2.6. Nakagami-m Fading Channel. If the signal amplitude follows a Nakagami distribution, then PDF of $\gamma$ follows a gamma PDF given by [11]

$$
f_{\gamma}(\gamma)=\left(\frac{m}{\bar{\gamma}}\right)^{m} \frac{\gamma^{m-1}}{\Gamma(m)} \exp \left(-\frac{m \gamma}{\bar{\gamma}}\right) ; \quad \gamma \geq 0,
$$

where $m$ is the Nakagami parameter. The average $P_{d}$ in the case of Nakagami- $m$ fading channel $\left(\bar{P}_{d, \mathrm{Nak}}\right)$ can be evaluated by substituting (14) in (7):

$$
\bar{P}_{d, \mathrm{Nak}}=\alpha\left[G_{1}+\beta \sum_{n=1}^{u-1} \frac{(\lambda / 2)^{n}}{2 n !}{ }_{1} F_{1}\left(m ; n+1 ; \frac{\lambda \bar{\gamma}}{2(m+\bar{\gamma})}\right)\right],
$$

where ${ }_{1} F_{1}(\cdot ; \cdot ; \cdot)$ is the confluent hyper geometric function (= $\Phi(\cdot ; \cdot ;))[24$, Section 9.2],

$$
\begin{gathered}
\alpha=\frac{1}{\Gamma(m) 2^{m-1}}\left(\frac{m}{\bar{\gamma}}\right)^{m}, \\
\beta=\Gamma(m)\left(\frac{2 \bar{\gamma}}{m+\bar{\gamma}}\right)^{m} \exp \left(-\frac{\lambda}{2}\right), \\
G_{1}=\frac{2^{m-1}(m-1) !}{(m / \bar{\gamma})^{m}} \frac{\bar{\gamma}}{m+\bar{\gamma}} \exp \left(-\frac{m \lambda}{2(m+\bar{\gamma})}\right) \\
\times\left[\left(1+\frac{m}{\bar{\gamma}}\right)\left(\frac{m}{m+\bar{\gamma}}\right)^{m-1} L_{m-1}\left(-\frac{\lambda \bar{\gamma}}{2(m+\bar{\gamma})}\right)\right. \\
\left.+\sum_{n=0}^{m-2}\left(\frac{m}{m+\bar{\gamma}}\right)^{n} L_{n}\left(-\frac{\lambda \bar{\gamma}}{2(m+\bar{\gamma})}\right)\right],
\end{gathered}
$$

where $L_{n}(\cdot)$ is the Laguerre polynomial of degree $n$ [24, Section 8.970]. We can obtain an alternative expression for $\bar{P}_{d \text {, Ray }}$ when setting $m=1$ in (17) and this expression is numerically equivalent to the one obtained in (11).

2.7. Weibull Fading Channel. In the Weibull fading model, the channel fading coefficient $h$ can be expressed as a function of the Gaussian in-phase $X$ and quadrature $Y$ elements of the multipath components $[25,26]$

$$
h=(X+j Y)^{2 / V} \text {, }
$$

where $j=\sqrt{-1}$.

Let $Z$ be the magnitude of $h$, that is, $Z=|h|$. If $R=\mid X+$ $j Y \mid$ is a Rayleigh distributed random variable, the Weibull distributed random variable can be obtained by transforming $R$ and using (18) as

$$
Z=R^{2 / V}
$$

From (19), the PDF of $Z$ can be given as

$$
f_{Z}(r)=\frac{V}{S} r^{V-1} \exp \left(-\frac{r^{V}}{S}\right)
$$

with $S=E\left(Z^{V}\right)$ and $E(\cdot)$ denoting the expectation. $V$ is the Weibull fading parameter expressing how severe the fading can be $(V>0)$ and $S$ is the average fading power. As $V$ increases, the effect of fading decreases, while for the special case of $V=2$, the Weibull PDF of $Z$ reduces to the Rayleigh PDF. For $V=1$ the Weibull PDF of $Z$ reduces to the well known negative exponential PDF.

The corresponding CDF of $Z$ can be expressed as [26]

$$
F_{Z}(r)=1-\exp \left(-\frac{r^{V}}{S}\right)
$$

In Weibull fading the instantaneous signal-to-noise ratio at a cognitive radio is given by [27]

$$
\gamma=Z^{2} \frac{E_{s}}{N_{01}} .
$$

It may be noted that the $n$th power of a Weibull distributed random variable with parameters $(V, S)$ is another Weibull distributed random variable with parameters $(V / n, S)$ [25]. Thus $\gamma$ is also a Weibull distributed random variable with parameters $\left(V / 2,(a \bar{\gamma})^{V / 2}\right)$ where $a=1 / \Gamma(1+2 / V)$. The PDF of $\gamma$ can then be derived from (14) by replacing $V$ with $V / 2$ and $S$ with $(a \bar{\gamma})^{V / 2}$ as [28]

$$
f_{\gamma}(\gamma)=\frac{V}{2(a \bar{\gamma})^{V / 2}}(\gamma)^{(V / 2-1)} \exp \left[-\left(\frac{\gamma}{a \bar{\gamma}}\right)^{V / 2}\right]
$$

where $\bar{\gamma}$ is the average SNR given as

$$
\bar{\gamma}=E\left(Z^{2}\right) \frac{E_{s}}{N_{01}}=S^{2 / V} \Gamma\left(1+\frac{2}{V}\right) \frac{E_{s}}{N_{01}} .
$$

Here $E\left(Z^{2}\right)$ is the second moment of $Z$ which can be obtained from the generalized expression for moments as [27]

$$
E\left(Z^{n}\right)=S^{n / V} \Gamma\left(1+\frac{n}{V}\right)
$$

where $n$ is a positive integer and $\Gamma(\cdot)$ is the Gamma function. The average $P_{d}$ in the case of a Weibull channel $\bar{P}_{d \text {,Weibu }}$ can be obtained analytically by substituting (23) in (7).

2.8. Hard Decision Fusion Rule (OR-Rule). Let $N$ denote the number of CR users sensing the PU. Each CR user makes its own local decision regarding the presence or absence of $\mathrm{PU}$ (i.e., $H_{1}$ or $H_{0}$ ) and forwards the binary decision ( 1 or 0$)$ to FC for data fusion as shown in Figure 2. The PU is located far away from all CR users. All the CR users receive the PU signal with the same local mean signal power. Further, the channels between CR users and FC are assumed to be ideal channels (noiseless). Assuming independent decisions, the fusion problem, where $k$-out of- $N$ CR users are needed for decision, can be described by binomial distribution based on Bernoulli trials where each trial represents the decision of each CR user. The generalized formula for overall probability of detection, $Q_{d}$, for the $k$-out of $N$ fusion rule is given by $[5,7]$

$$
Q_{d}=\sum_{l=k}^{N}\left(\begin{array}{c}
N \\
l
\end{array}\right) \bar{P}_{d}^{l}\left(1-\bar{P}_{d}\right)^{N-1}
$$




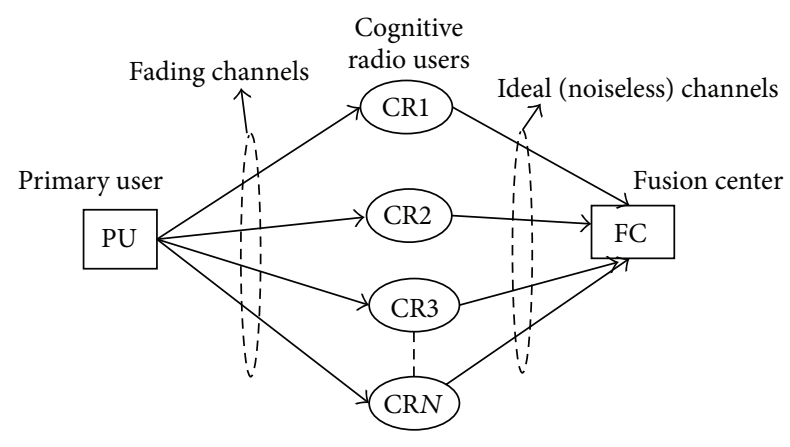

FIgURE 2: Cooperative spectrum sensing system.

where $P_{d}$ is the probability of detection for each individual CR user as defined by generalized (5) and (7).

The OR-logic fusion rule (i.e., 1-out of $-N$ rule) can be evaluated by setting $k=1$ in (26):

$$
\begin{aligned}
Q_{d, \mathrm{OR}} & =\sum_{l=1}^{N}\left(\begin{array}{c}
N \\
l
\end{array}\right) \bar{P}_{d}^{l}\left(1-\bar{P}_{d}\right)^{N-1} \\
& =1-\left.\left(\begin{array}{c}
N \\
l
\end{array}\right) \bar{P}_{d}^{l}\left(1-\bar{P}_{d}\right)^{N-1}\right|_{l=0}=1-\left(1-\bar{P}_{d}\right)^{N} .
\end{aligned}
$$

Equation (27) can be rewritten as

$$
\begin{gathered}
Q_{d}=1-\prod_{i=1}^{N}\left(1-\bar{P}_{d}\right), \\
Q_{m}=1-Q_{d}, \\
Q_{f}=1-\prod_{i=1}^{N}\left(1-P_{f}\right) .
\end{gathered}
$$

In conventional nonweighted fusion (hard decision fusion), the noise, fading statistics, and average SNR in sensing channel are the same for each CR user. But in a real cognitive radio system this may not be true, that is, each CR experiences different fading statistics. Under this assumption two weighting schemes are proposed [18] and explained in detail in the following subsections.

2.9. Channel Preference Based Weighting Scheme. In this paper, we assume that each CR user experiences different channel status with the PU. Though we assume fading in sensing channel, the reporting channel is assumed to be ideal as FC is considered to be situated near to CRs [6-9]. The difference in channel status of the CR users necessitates considering some of the CR users more prior in making the decision about the PU than the other CR users. Assuming that FC knows each sensing channel status and each CR user experiences different channel status, we can weight their decisions based on following certain weighting algorithm. Assume that we generate a decision at FC which is equivalent to the decision of the worse channel status CR. Then replace it with the decision of the best channel status CR. These two generation and replacement operations are done in a way to hold two conditions: the total number of CR users must not change and decision independency is maintained. Equation (28) can be modified as

$$
Q_{d}=1-\left\{\left(1-\bar{P}_{d}^{1}\right)\left(1-\bar{P}_{d}^{2}\right) \cdots\left(1-\bar{P}_{d}^{N}\right)\right\} .
$$

Let us consider an example that $\mathrm{CR}_{1}$ user experiences a better channel with PU, say AWGN channel, and other CRs experience the same worst channel, say Rayleigh faded channel. The decision of $\mathrm{CR}_{1}$ may be considered as decision of $k$ CRs instead of the one and remaining $(N-1)$ CRs as decision of $(N-k)$. This could be done by modifying (30) as [18]

$$
Q_{d}=1-\left\{\left(1-\widetilde{P}_{d}\right)^{k}\left(1-\bar{P}_{d}\right)^{N-k}\right\},
$$

where $\widetilde{P}_{d}$ is the probability of detection at $k$ number of CR users in AWGN channel and $\bar{P}_{d}$ is the probability of detection at $N-k$ number of $\mathrm{CR}$ users in faded channel. This algorithm is named as $k$-Gaussian and $N-k$ faded algorithm.

2.10. Decision Statistic Based Weighting Scheme. In a real cognitive radio system different CR users may have the same opinion about the presence or the absence of the PU. But decision statistic value need not be the same for all CR users. Some may have high decision statistic value, while others may have low decision statistic value. Because of this difference, the dependability on the decisions of different CRs should be different. This means that the final decision must be taken according to the preference of some CR users over the others. Let us assume that all CR users are having the same detection threshold $\lambda$ value and let us define a reliability factor $\left(R_{f}\right), R_{f}$ as the difference between the decision statistic value $Y$ and the detection threshold value as given in [18]

$$
R_{f}=Y-\lambda .
$$

The reliability factor must be included in the probability of detection. For example consider 4-CR users in which one $\mathrm{CR}$ user (say $\mathrm{CR}_{1}$ user) is having highest statistical value of $Y=1.4 \lambda$ and others are having the same value of $Y=1.2 \lambda$. Then the reliability factor for this network will be evaluated from the value of the decision statistic obtained from $\mathrm{CR}_{1}$ user to give $R_{f}=0.4$. The reliability factor is multiplied with probability of missed detection of $\mathrm{CR}_{1}$ user, $\bar{P}_{m 1}$ at FC; $Q_{d}$ can be expressed as [18]

$$
\begin{gathered}
Q_{d}=1-\left[\left\{R_{f} * \bar{P}_{m 1}\right\} \bar{P}_{m 2} \ldots \bar{P}_{m N}\right] ; \\
\bar{P}_{m 1,2, \ldots, N}=1-\bar{P}_{d 1,2, \ldots, N} .
\end{gathered}
$$

\section{Simulation Model}

The simulation is developed in MATLAB. To obtain the fading channel power distribution, one can rely on the amplitude/envelope distribution. Let us assume that each multipath component (MPC) obeys an instantaneous fading 
TABLE 1: Type of fading and generation.

\begin{tabular}{|c|c|}
\hline Tape of fading coefficient $(h)$ & Generation \\
\hline Log-normal shadowing (parameter, $\sigma_{\mathrm{dB}}$ ) & $\begin{array}{c}X \sim N\left(0, \sigma^{2}\right) \text { where } \sigma=0.1 \ln (10) \sigma_{\mathrm{dB}} \\
h=e^{X}\end{array}$ \\
\hline Hoyt (parameter, $q$ ) & $\begin{array}{c}X \sim N\left(0, q^{2} / 1+q^{2}\right) ; Y \sim N \\
h=\sqrt{X^{2}+Y^{2}}\end{array}$ \\
\hline Rayleigh & $\begin{array}{c}X \sim N(0,1 / 2) ; Y \sim N(0,1 / 2) \\
h=\sqrt{X^{2}+Y^{2}}\end{array}$ \\
\hline \multirow[t]{2}{*}{ Rician (parameter, $K$ ) } & $X \sim N\left(s^{\prime}, \sigma^{2}\right) ; Y \sim N\left(0, \sigma^{2}\right)$, where $s^{\prime}=\sqrt{K /(K+1)}$ and $\sigma=1 / \sqrt{2(1+K)}$ \\
\hline & $h=\sqrt{X^{2}+Y^{2}}$ \\
\hline Nakagami- $m$ (parameter, $m$ ) & $\begin{array}{c}X \sim \operatorname{gamma}(r, s) ; \text { where } r=m ; s=1 / m ; \\
h=\sqrt{X}\end{array}$ \\
\hline Weibull (parameter, $V$ ) & $\begin{array}{c}X \sim N(0,1 / 2) ; Y \sim N(0,1 / 2) \\
h=\left(X^{2}+Y^{2}\right)^{2 / V}\end{array}$ \\
\hline
\end{tabular}

amplitude/envelope of $a=|h|$ with PDF $P_{a}(a)$. The instantaneous power of the said fading channel is thus given as $g=a^{2}$ with PDF $P_{g}(g)$; its average $\bar{g}=E\{g\}=E\left\{a^{2}\right\}$ is often normalized to unity, that is, $\bar{g}=1$ using a simple PDF transformation; one can relate the PDF of the channel power with the one of the envelope which is given by [18]

$$
P_{g}(g)=\frac{1}{2 \sqrt{g}} P_{a}(\sqrt{g}) .
$$

And inversely

$$
P_{a}(a)=2 a \cdot P_{g}\left(a^{2}\right)
$$

The simulation is carried out according to the following steps to verify the analytical framework introduced in the previous section.

(1) Equally likely hypothesis $H \in\left\{H_{0}, H_{1}\right\}$ and primary user (PU) signal $s(t)$ are generated using uniform random variable generator.

(2) The AWGN signal $n(t)$ with zero mean variance $N_{01} W$ is generated using Gaussian random variable, where $N_{01}=E_{s} / \bar{\gamma}$. The sensing channel coefficient $h_{i}$ at $i$ th CR user in case of Hoyt, Rayleigh, Rician, and Weibull fading is generated using two Gaussian random variables. The Nakagami- $m$ fading channel coefficient can be generated using Gamma distribution and Log-normal shadowing coefficient can be generated as explained in Section 2.2. In case of non-fading environment (pure AWGN), $h_{i}=1$. The way of generating several types of fading coefficients (Log-normal shadowing, Hoyt, Rayleigh, Rician, Nakagami- $m$, and Weibull) is shown in Table 1 .

(3) The received signal at $i$ th CR user is $x_{i}(t)=h_{i} s(t)+$ $n_{i}(t)$ for true hypothesis $H_{1}$ and $x_{i}(t)=n_{i}(t)$ for true hypothesis $H_{0}$.

(4) The received signal at $i$ th CR user $x_{i}(t)$ is given as the input to band pass filter (BPF) and its output is

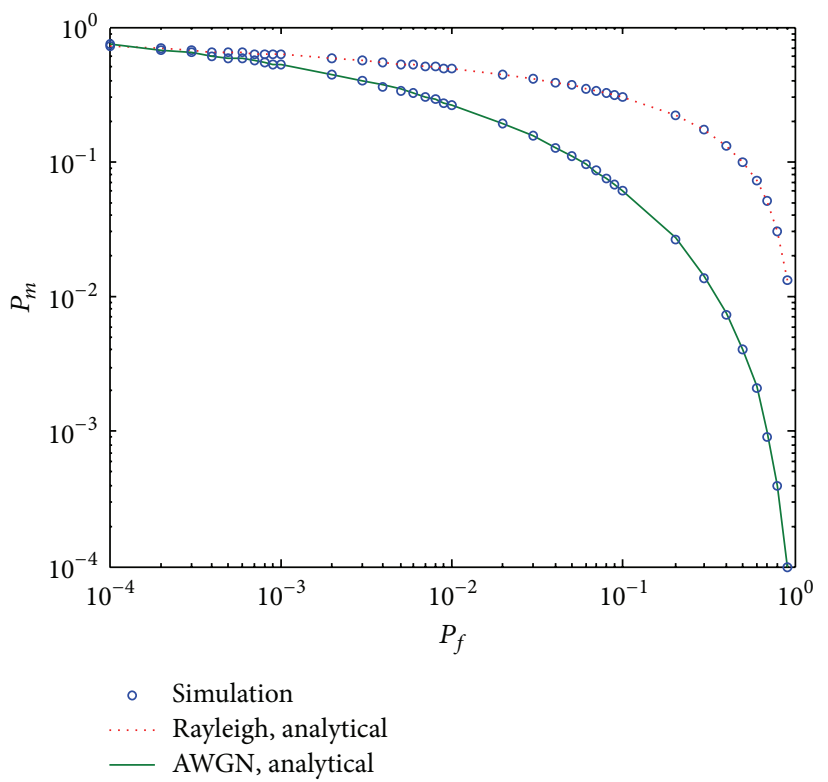

FIGURE 3: CROC curves for single CR user based spectrum sensing in AWGN and Rayleigh channel [6].

squared (i.e. $\left.x_{i}^{2}(t)\right)$ using squaring device and passed through an integrator. The output of the integrator is obtained as $Y_{i}=\sum_{j=1}^{2 m} n_{i, j}^{\prime 2}($ from (4)).

(5) A predefined detection threshold $\lambda$ is set at ED depending on chosen $P_{f}$ (from (6)). The ED of $i$ th CR user compares decision statistic at $i$ th CR user $Y_{i}$ with its detection threshold $\lambda$ and takes a hard binary decision (1 or 0 ) about PU.

(6) The steps 1 to 6 have been repeated a large number of times and then the average value of $P_{d}$ and $P_{m}$ has been estimated.

(7) The overall probability of detection $Q_{d}$ can be estimated by substituting average value of $P_{d}$ in (28), (31), and (33). 


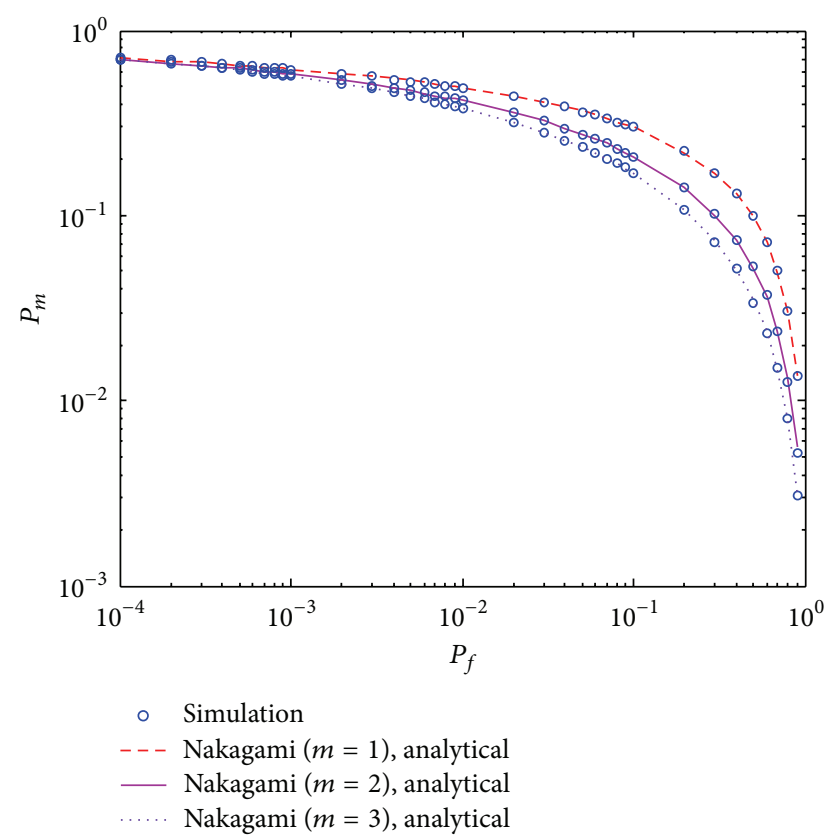

Figure 4: Effect of Nakagami fading parameter $(m)$ on single CR user based spectrum sensing.

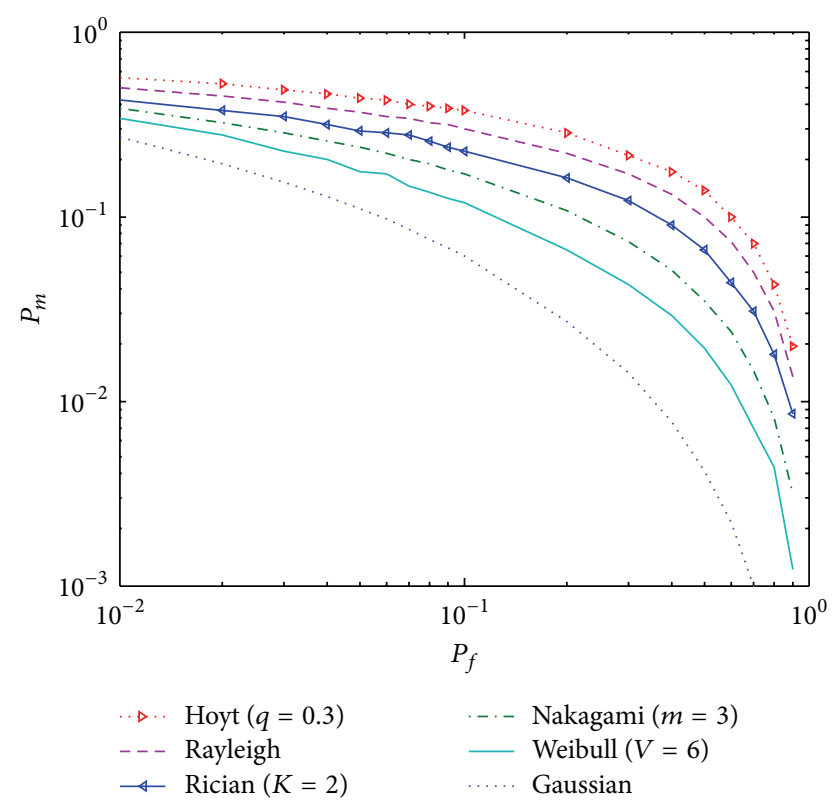

FIGURE 5: CROC curves for single CR user based spectrum sensing in different fading channels.

\section{Results and Discussions}

Using the steps as mentioned in Section 3, we evaluated the performance of two different weighting schemes (channel preference based and decision statistic value based weighting schemes) in non-fading (AWGN) channel, in several fading channels such as Hoyt, Rayleigh, Rician, Nakagami-m, Weibull, and log-normal shadowing channel. The overall probabilities of missed detection $\left(Q_{m}\right)$ and false alarm $\left(Q_{f}\right)$

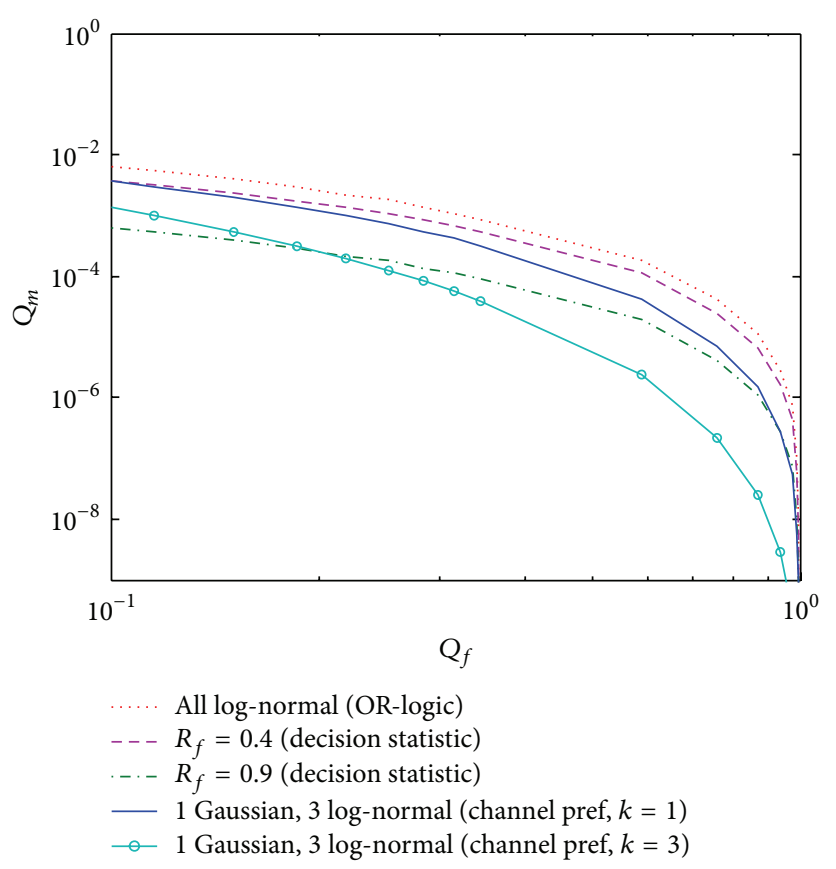

FIGURE 6: CROC curves for 4 CR users using decision statistic and channel preference based weighting schemes in log-normal shadowing $(2 \mathrm{~dB})$ channel.

at FC are evaluated under different channel conditions. The performance of CSS with OR logic fusion is also shown for comparison purpose. Simulation results are obtained with the following parameters, the number of CR users is $N=4$, time-bandwidth product is $u=5$, and the average sensing channel SNR is $\bar{\gamma}=10 \mathrm{~dB}$. The weighting factor of channel preference based scheme, $k$, is considered as 1 and 3 and weighting factor of decision statistic based weighting scheme, $R_{f}$ is considered as 0.4 and 0.9 . Figures 3 to 5 are results of spectrum sensing using a single CR user and Figures 6 to 11 are results of cooperative spectrums sensing using weighting schemes.

Figure 3 shows the variation of missed detection probability $\left(P_{m}\right)$ as a function of the false alarm probability $\left(P_{f}\right)$ for single CR user based spectrum sensing. The performance is evaluated in AWGN and Rayleigh fading channel using analytical expressions and simulation. The AWGN curve is provided for comparison. As $P_{f}$ increases, $P_{m}$ decreases more rapidly for AWGN channel than for Rayleigh fading channel. Our simulation results match with the theoretical results.

In Figure 4, the effect of Nakagami parameter $(m)$ on the $P_{m}$ of single CR user based spectrum sensing is shown. As the Nakagami parameter $m$ increases from 1 to 3 , the probability of missed detection $P_{m}$ decreases which is intuitively true as higher $m$ indicates a less sever fading. For example, for $P_{f}=$ 0.001 , the $P_{m}$ values for $m=1,2$, and 3 are $0.6192,0.5776$, and 0.5638 , respectively.

Figure 5 shows complementary ROC curves for spectrum sensing with single CR user in the presence of Hoyt, Rayleigh, Rician, Nakagami- $m$, and Weibull fading channels. Hoyt, Rician, Nakagami- $m$, and Weibull fading parameters are 


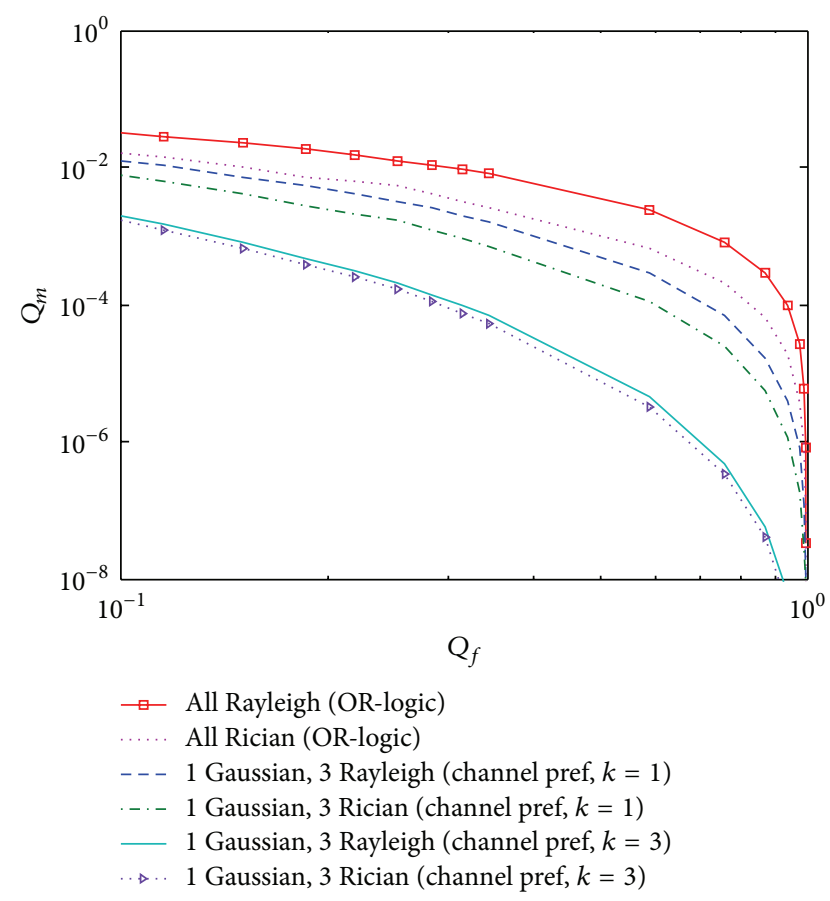

FIGURE 7: CROC curves for 4 CR users using channel preference based weighting scheme in Rayleigh and Rician $(K=2)$ fading channels.

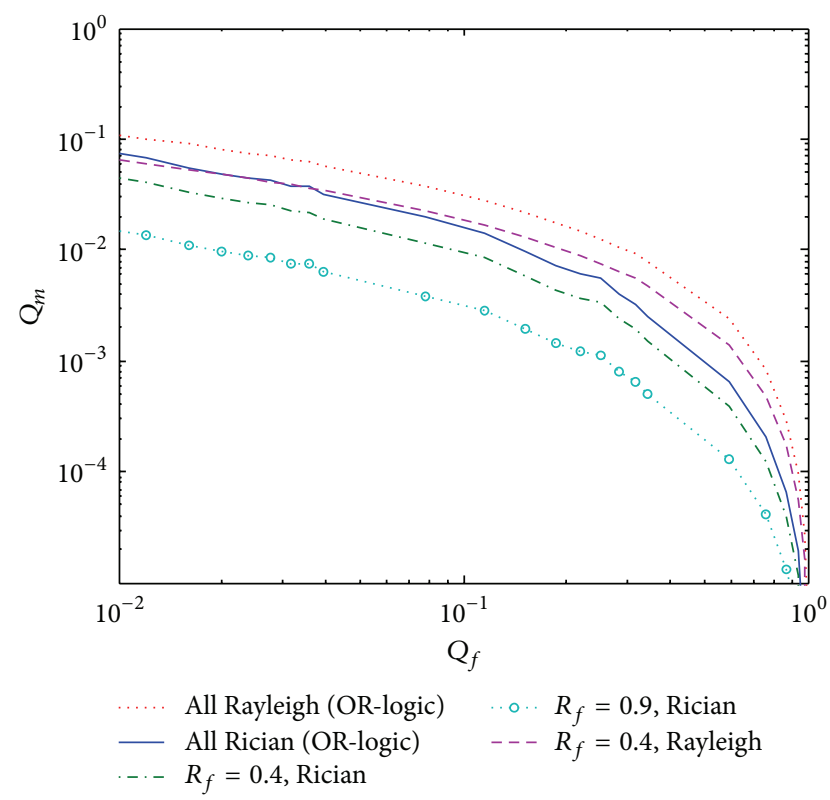

FIGURE 8: CROC curves for 4 CR users using decision statistic based weighting scheme in Rayleigh and Rician $(K=2)$ fading channels.

assumed to be $q=0.3, K=2, m=3$, and $V=6$, respectively. Comparing the AWGN curve with those corresponding to fading, we observe that spectrum sensing performance degrades in the presence of fading. The performance of energy detector is the best in Weibull fading channel among all fading channels.

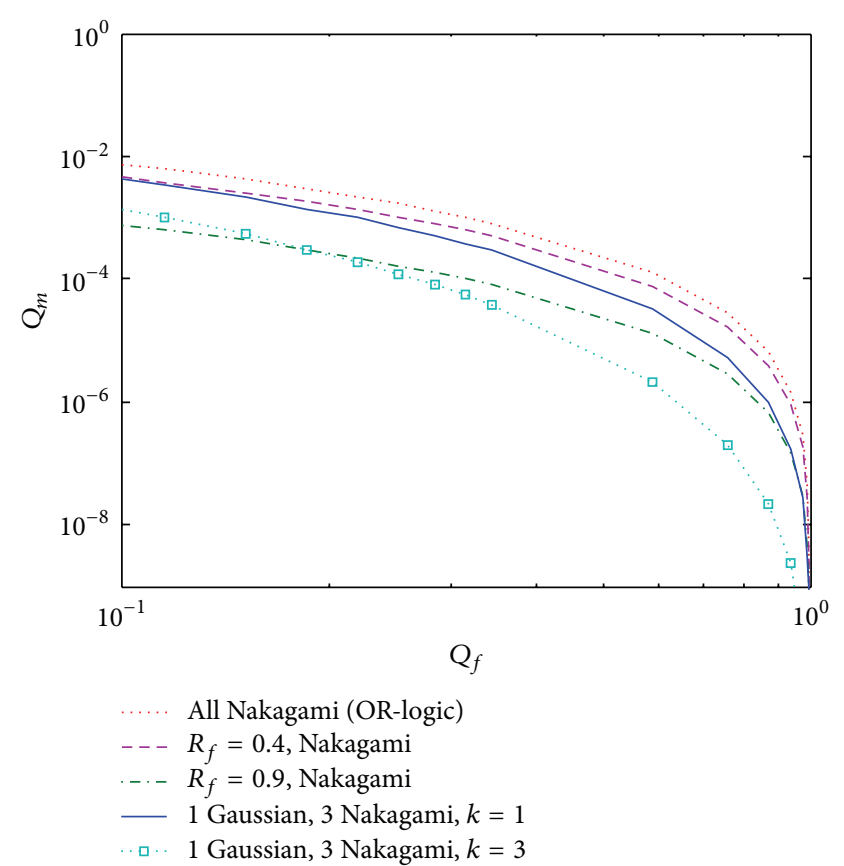

FIGURE 9: CROC curves for 4 CR users using decision statistic and channel preference based weighting schemes in Nakagami $(m=3)$ fading channel.

Now we show the results on spectrum sensing based on weighting schemes.

In Figure 6, the performance comparison between cooperative spectrum sensing using two weighting schemes (decision statistic based and channel preference based) has been evaluated in the presence of log-normal shadowing. Two different values of weighting factor for the channel preference based weighting scheme $(k=1$ and 3$)$ and two different values of weighting factor for decision statistic based weighting scheme $\left(R_{f}=0.4\right.$ and 0.9$)$ have been considered for this figure. The performance of conventional CSS with OR-logic fusion is also shown for comparison purpose. We observed that the performance of CSS with both weighting schemes is better than the performance of CSS with OR-logic fusion. It is observed that $Q_{m}$ decreases with increase in any one of $Q_{f}, k$, and $R_{f}$. For example, at $Q_{f}=0.25$, when $k$ increases from 1 to $3, Q_{m}$ decreases from 0.0007 to 0.0001 . Similarly, when $R_{f}$ increases from 0.4 to $0.9, Q_{m}$ decreases from 0.0011 to 0.0002 for the same value of $Q_{f}$.

Figures 7 and 8 show the performance of CSS with channel preference based and decision statistic based weighting schemes, respectively. The performance is evaluated in both Rayleigh and Rician fading channels. It is seen from Figure 7 that as $k$ as well as $Q_{f}$ increases, $Q_{m}$ decreases for both fading channels. For example, for $Q_{f}=0.25$, as $k$ increases from 1 to $3, Q_{m}$ decreases from 0.0031 to 0.0002 for Rayleigh fading channel and $Q_{m}$ decreases from 0.0017 to 0.0002 for Rician fading channel as shown in Figure 7. Similarly as $R_{f}$ decreases from 0.4 to $0.9, Q_{m}$ decreases from 0.0034 to 0.0011 for the same value of $Q_{f}$ for Rician fading channel as shown in Figure 8. We observed that the performance of CSS in 


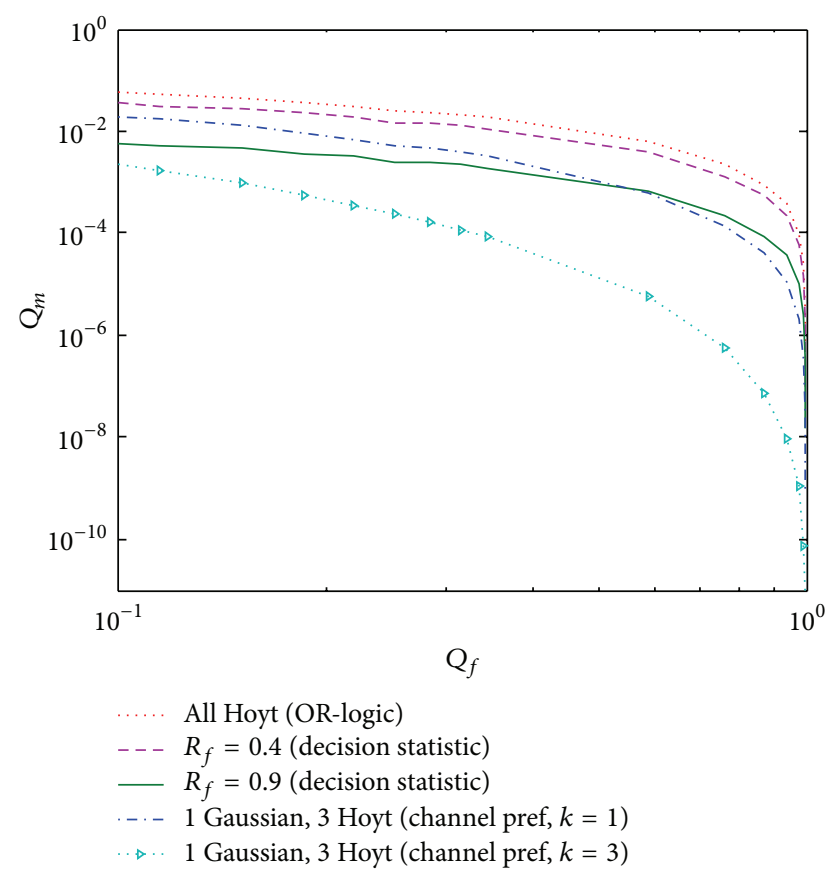

FIGURE 10: CROC curves for $4 \mathrm{CR}$ users using decision statistic and channel preference based weighting schemes in Hoyt $(q=0.3)$ fading channel.

Rician fading channel is better than the performance of CSS in Rayleigh fading channel for the same value of $k$ and $R_{f}$ due to presence of line of sight (LOS) signal component. The performance of CSS with OR-logic fusion is provided for comparison purpose.

Figures 9 and 10 show the performance comparison between two weighting schemes (decision statistic based weighting scheme and channel preference based weighting scheme) in Nakagami- $m$ and Hoyt fading channels, respectively. Two different values of weighting factors $k$ ( 1 and 3 ) and $R_{f}(0.4$ and 0.9$)$ have been considered for these figures. We observe from both figures that $Q_{m}$ decreases with increase in any one of $Q_{f}, k$, and $R_{f}$. For example, at $Q_{f}=0.25, Q_{m}$ decreases from 0.0007 to 0.0001 and from 0.0010 to 0.0002 as $k$ increases from 1 to 3 and as $R_{f}$ increases from 0.4 to 0.9 , respectively (in Figure 9). Similarly, in Figure 10, $Q_{m}$ decreases from 0.0052 to 0.0002 and from 0.0147 to 0.0025 when $k$ increases from 1 to 3 and $R_{f}$ increases from 0.4 to 0.9 , respectively. The performance of CSS with channel preference based weighting scheme, for $k=3$, is better than the performance with decision statistic based weighting scheme for $R_{f}=0.9$ as seen in Figures 9 and 10. The performance of CSS with OR-logic fusion is also shown for comparison purpose. As we observe from the above curves in Figures 9 and 10, once again both the weighting schemes based fusion outperform OR-logic fusion.

In Figure 11, the performance comparison between decision statistic based weighting scheme and channel preference based weighting scheme in Weibull fading channel is shown. As in earlier case, two different values of weighting factors $k$ ( 1 and 3 ) and $R_{f}(0.4$ and 0.9$)$ have also been considered for this

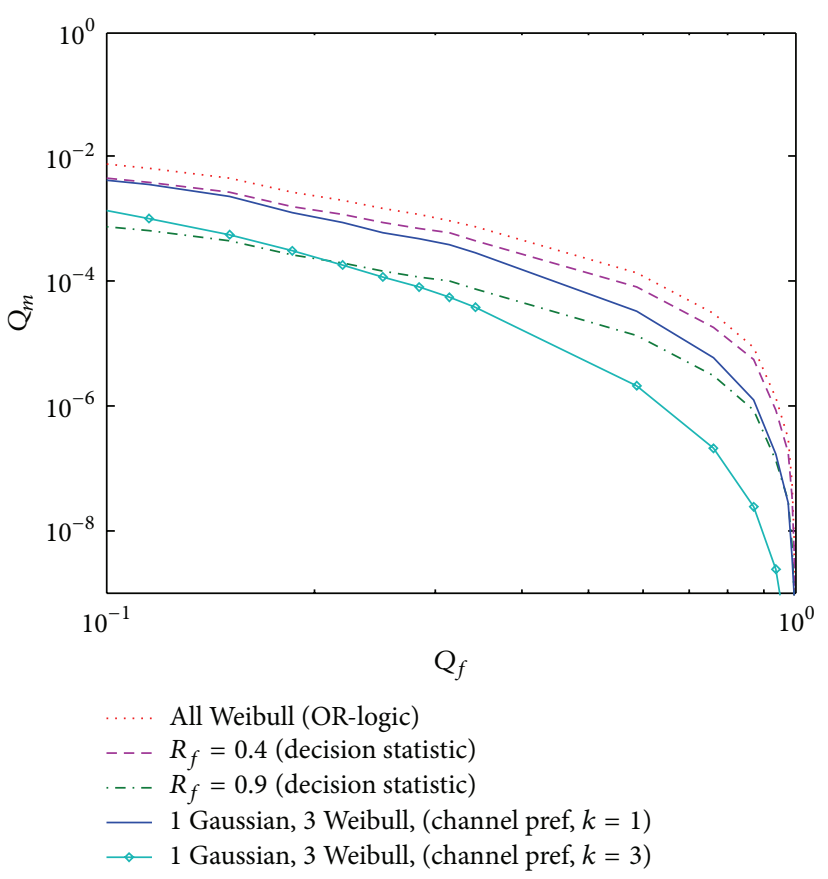

FIGURE 11: CROC curves for 4 CR users using decision statistic and channel preference based weighting schemes in Weibull $(V=4)$ fading channel.

figure. As any one of $Q_{f}, k$, and $R_{f}$ increases, $Q_{m}$ decreases. For example, for $Q_{f}=0.25, Q_{m}$ decreases from 0.0007 to 0.0001 and from 0.0003 to 0.0001 as $k$ increases from 1 to 3 and $R_{f}$ increases from 0.4 to 0.9 , respectively. The performance of CSS with channel preference based weighting scheme, for $k=$ 3 , is better than the performance with decision statistic based weighting scheme for $R_{f}=0.9$. The performance of CSS with OR-logic fusion is also shown for comparison in this figure. Weighting schemes guarantee a better performance than ORlogic based fusion in conventional cooperative spectrum sensing scenario.

Figure 12 shows the impact of several fading channels such as Hoyt, Rayleigh, Rician, Nakagami- $m$, and Weibull fading on the performance of CSS using channel preference $(k=2)$ based weighting scheme. Hoyt, Rician, Nakagami- $m$ and Weibull fading parameters are assumed to be $q=0.3$, $K=2, m=3$, and $V=6$, respectively. The performance of CSS in Weibull fading is better than other fading channels for the same value of $k$. For example, for $Q_{f}=0.25$ and $k=2$, the values of $Q_{m}$ for Hoyt, Rayleigh, Rician, Nakagami- $m$, and Weibull channels are $1.1 \times 10^{-3}, 8 \times 10^{-4}, 5 \times 10^{-4}, 3 \times 10^{-4}$, and $1 \times 10^{-4}$, respectively.

\section{Conclusions}

We have investigated the performance of single CR user based spectrum sensing, considering several fading in sensing channels such as Hoyt, Rayleigh, Rician, Nakagami- $m$, and Weibull. We have also evaluated the performance of cooperative spectrum sensing (CSS) using OR-logic fusion and two different weighting schemes such as channel preference 


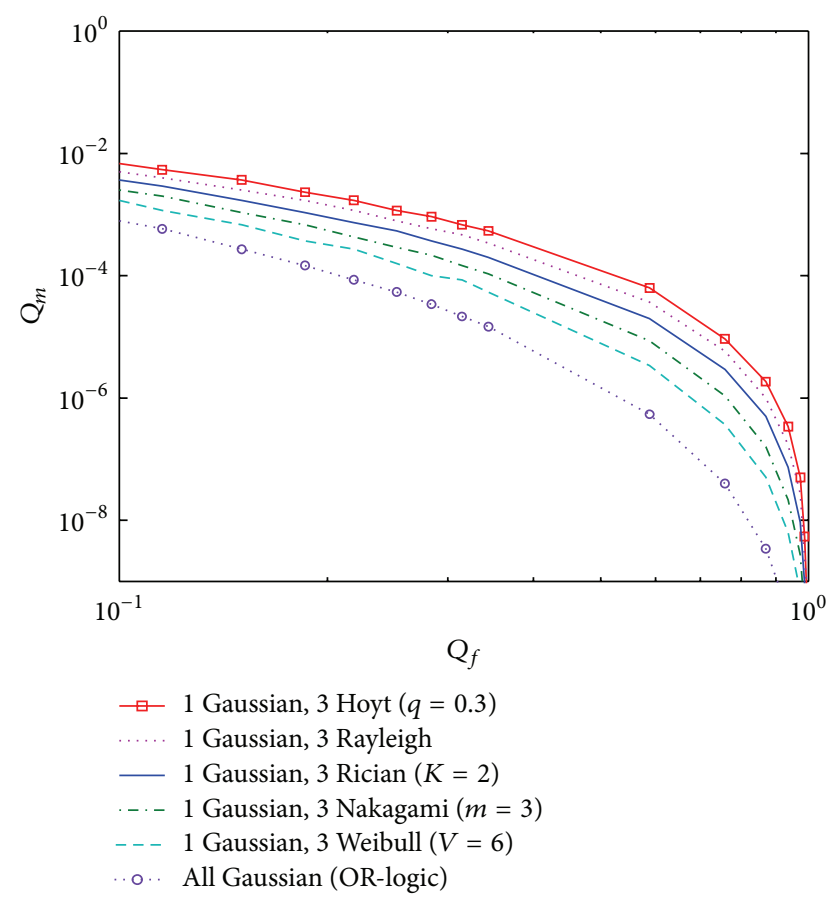

FIGURE 12: CROC curves for $4 \mathrm{CR}$ users using channel preference based weighting scheme $(K=2)$ in different fading channels.

based weighting scheme and decision statistic based weighting scheme. The performance of CSS with channel preference based weighting scheme for higher value of $k$ is found to be better than that with decision statistic based weighting scheme for maximum value of $R_{f}$ Performance comparison of CSS with weighting schemes among fading channels has been evaluated and the performance of CSS in Weibull fading channel outperforms that of any other fading channel considered in this paper. The performances with both weighting schemes outperform OR-logic based fusion. The overall probability of missed detection decreases with increase in overall probability of false alarm and weighting factors $(k$ and $R_{f}$ ). The above study is useful in designing weighted based cooperative spectrum sensing network under several fading environments in sensing channels.

\section{References}

[1] S. Haykin, "Cognitive radio: brain-empowered wireless communications," IEEE Journal on Selected Areas in Communications, vol. 23, no. 2, pp. 201-220, 2005.

[2] F. F. Digham, M.-S. Alouini, and M. K. Simon, "On the energy detection of unknown signals over fading channels," in Proceedings of the International Conference on Communications (ICC '03), vol. 5, pp. 3575-3579, Anchorage, Alaska, USA, May 2003.

[3] H. Urkowitz, "Energy detection of unknown deterministic signals," in Proceedings of IEEE, vol. 55, pp. 523-231, April 1967.

[4] D. Cabric, S. M. Mishra, and R. W. Brodersen, "Implementation issues in spectrum sensing for cognitive radios," in Conference Record of the 38th Asilomar Conference on Signals, Systems and Computers, pp. 772-776, Asilomar, Calif, USA, November 2004.
[5] S. Nallagonda, S. Suraparaju, S. D. Roy, and S. Kundu, "Performance of energy detection based spectrum sensing in fading channels," in Proceedings of the 2nd International Conference on Computer and Communication Technology (ICCCT'11), pp. 575580, Allahabad, India, September 2011.

[6] A. Ghasemi and E. S. Sousa, "Collaborative spectrum sensing for opportunistic access in fading environments," in Proceedings of the 1st IEEE International Symposium on New Frontiers in Dynamic Spectrum Access Networks (DySPAN '05), pp. 131-136, Baltimore, MD, USA, November 2005.

[7] J. Duan and Y. Li, "Performance analysis of cooperative spectrum sensing in different fading channels," in Proceedings of the 2nd International Conference on Computer Engineering and Technology (ICCET '10), pp. V364-V368, Chengdu, China, June 2010.

[8] S. Nallagonda, A. Chndra, S. D. Roy, and S. Kundu, "Performance of cooperative spectrum sensing in Hoyt fading channel under Hard decision Fusion rules," in Proceedings of the 5th IEEE International Conference on Computers and Devices for Communication (CODEC '12), Kolkata, India, December 2012.

[9] S. Nallagonda, S. D. Roy, and S. Kundu, "Performance of cooperative spectrum sensing in Fading Channels," in Proceedings of the IEEE International Conference on Recent Advances in Information Technology (RAIT '12), ISM, Dhanbad, India, March 2012.

[10] S. Nallagonda, S. D. Roy, and S. Kundu, "Performance of Cooperative spectrum sensing in Log-normal Shadowing and Fading under Fusion rules," International Journal of Energy, Information and Communications, Science \& Engineering Research Support Center, vol. 3, no. 3, pp. 15-28, 2012.

[11] S. Nallagonda, S. D. Roy, and S. Kundu, "Performance of cooperative spectrum sensing in Rician and Weibull fading channels," in Proceedings of the Annual IEEE India Conference: Engineering Sustainable Solutions (INDICON '11), Hyderabad, India, December 2011.

[12] R. S. Hoyt, "Probability functions for the modulus and angle of the normal complex variate," Bell System Technical Journal, vol. 26, pp. 318-359, 1947.

[13] A. Chandra, C. Bose, and M. K. Bose, "Performance of noncoherent MFSK with selection and switched diversity over hoyt fading channel," Wireless Personal Communications, vol. 68, no. 2, pp. 379-399, 2013.

[14] M. K. Simon and M.-S. Alouini, Digital Communication Over Fading Channels, Wiley, New Jersey, NJ, USA, 2nd edition, 2004.

[15] H. Hashemi, "Indoor radio propagation channel," Proceedings of the IEEE, vol. 81, no. 7, pp. 943-968, 1993.

[16] N. S. Adawi, "Coverage prediction for mobile radio systems operating in the $800 / 900 \mathrm{MHz}$ frequency range," IEEE Transactions on Vehicular Technology, vol. 37, no. 1, pp. 3-72, 1988.

[17] S. Kyperountas, N. Correal, Q. Shi, and Z. Ye, "Performance analysis of cooperative spectrum sensing in Suzuki fading channels," in Proceedings of the 2nd IEEE International Conference on Cognitive Radio Oriented Wireless Networks and Communications (CrownCom '07), pp. 428-432, Orlando, Fla, USA, August 2007.

[18] M. R. Alrabeiah and I. S. Alnomay, "A cooperative weighted discriminator energy detector technique in fading environment," World Academy of Science, Engineering and Technology, vol. 80, pp. 397-400, 2011.

[19] S.-W. Wu, J.-K. Zhu, L. Qiu, and M. Zhao, "SNR-based weighted cooperative spectrum sensing in cognitive radio networks," 
Journal of China Universities of Posts and Telecommunications, vol. 17, no. 2, pp. 1-7, 2010.

[20] L. Khalid and A. Anpalagan, "A weighted fusion scheme for cooperative spectrum sensing based on past decisions," in Proceedings of the IEEE 22nd International Symposium on Personal, Indoor and Mobile Radio Communications (PIMRC '11), pp. 354358, Toronto, Canada, September 2011.

[21] J. Xue, L. Li, S. Chen, and L. Huang, "Exponential weighted collaborative Spectrum Sensing Based on SNR," in Proceedings of the International Conference on Multimedia Technology (ICMT '10), pp. 1-4, Ningbo, China, October 2010.

[22] S. Nallagonda, S. K. Bandari, S. D. Roy, and S. Kundu, "Performance of Weighted Cooperative spectrum sensing Schemes in Fading Channels," in Proceedings of the IEEE International Conference on Communications, Devices and Intelligent Systems (CODIS '12), Jadavpur University, Kolkata, India, December 2012.

[23] I. S. Gradshteyn and I. M. Ryzhik, Table of Integrals, Series, and Products, Academic Press, 5th edition, 1994.

[24] A. H. Nuttall, "Some integrals involving the QM function," IEEE Transactions on Information Theory, vol. 21, no. 1, pp. 95-96, 1975.

[25] M. Lupupa and M. E. Dlodlo, "Performance analysis of transmit antenna selection in Weibull fading channel," in Proceedings of the 9th IEEE AFRICON Conference, pp. 1-6, Nairobi, Kenya, September 2009.

[26] N. C. Sagias and G. K. Karagiannidis, "Gaussian class multivariate Weibull distributions: theory and applications in fading channels," IEEE Transactions on Information Theory, vol. 51, no. 10, pp. 3608-3619, 2005.

[27] N. C. Sagias and G. S. Tombras, "On the cascaded Weibull fading channel model," Journal of the Franklin Institute, vol. 344, no. 1, pp. 1-11, 2007.

[28] N. C. Sagias, D. A. Zogas, G. K. Karagiannidis, and G. S. Tombras, "Channel capacity and second-order statistics in weibull fading," IEEE Communications Letters, vol. 8, no. 6, pp. 377-379, 2004. 

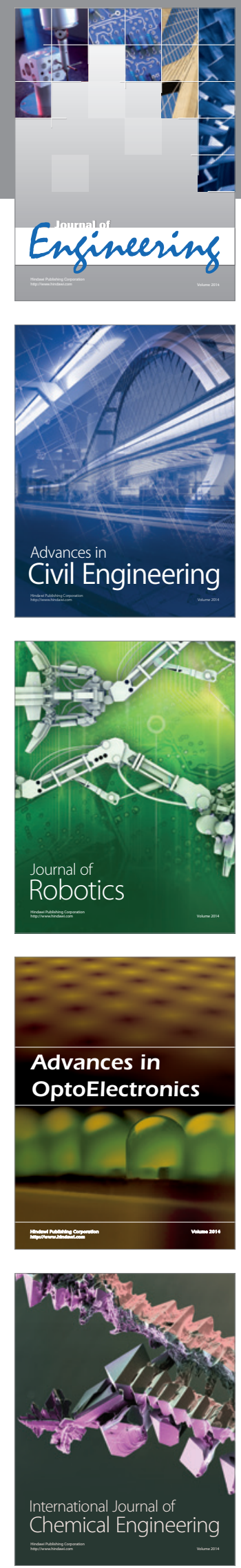

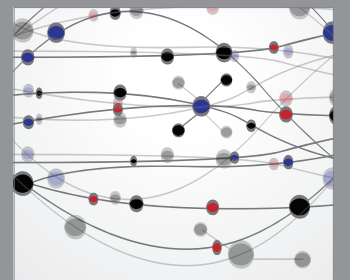

The Scientific World Journal
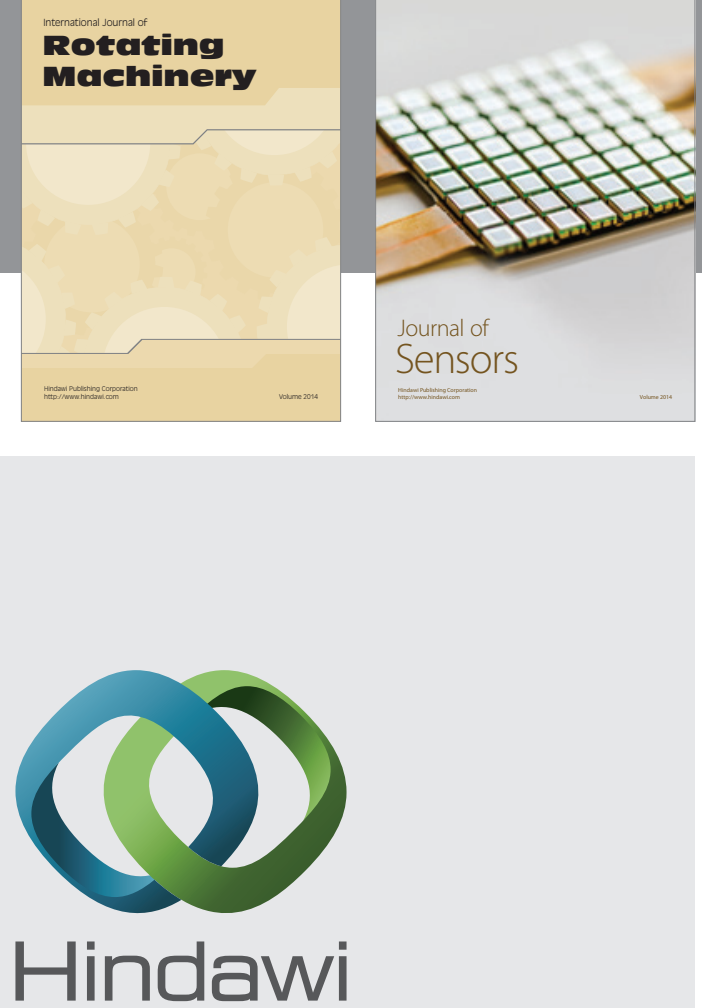

Submit your manuscripts at http://www.hindawi.com
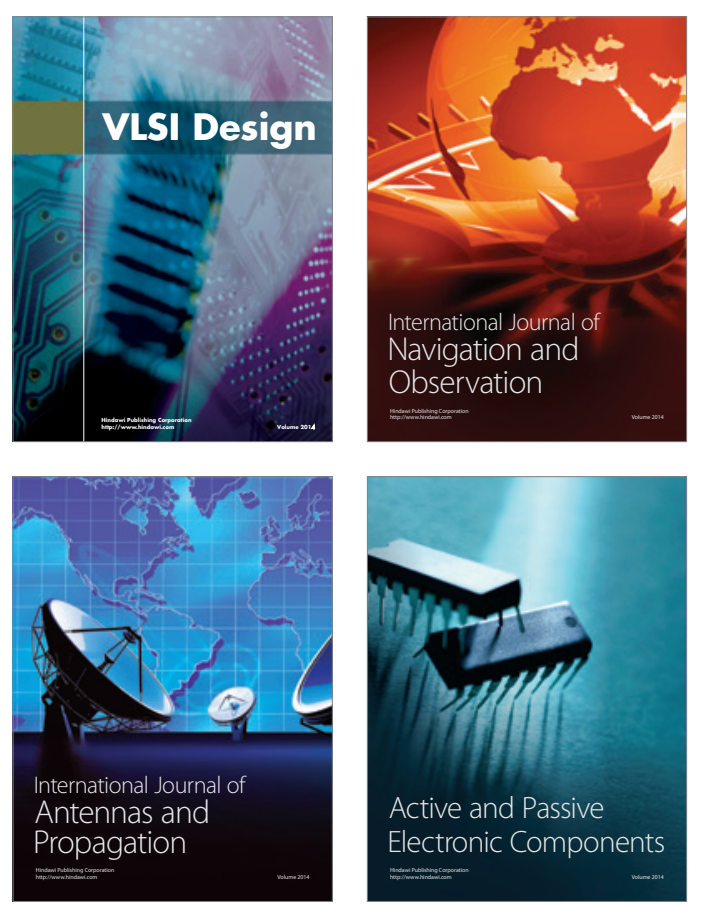
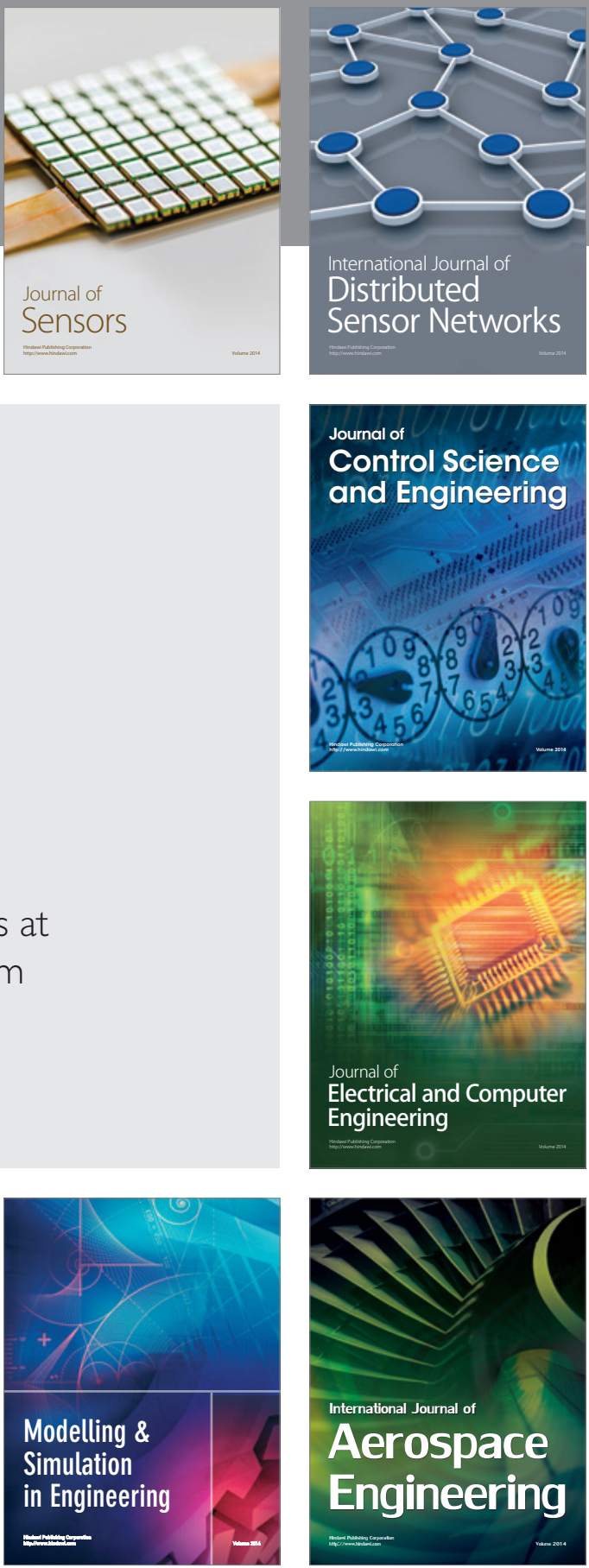

Journal of

Control Science

and Engineering
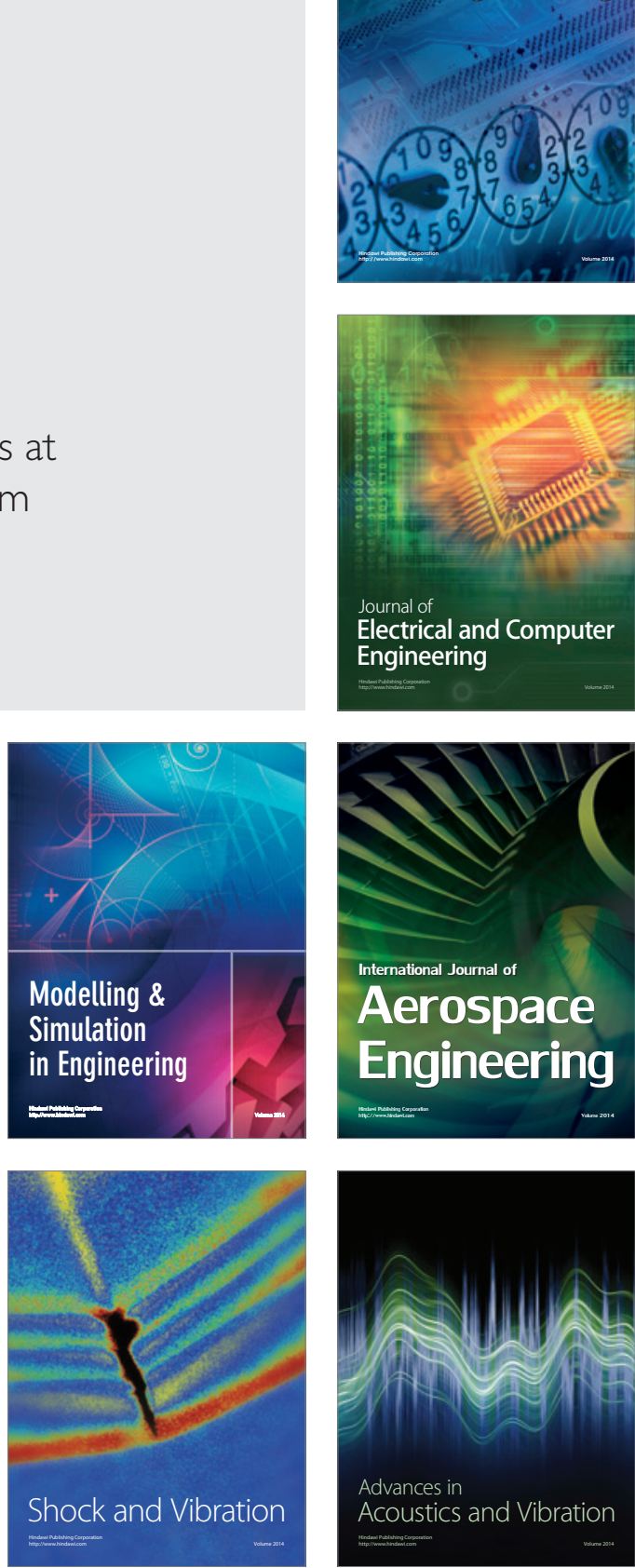